\title{
NATURALEZA Y PRODUCCIÓN: HACIA LA SUSTANCIALIDAD DE LA OBRA DE ARTE EN ARISTÓTELES
}

Cecilia Sabido*

\begin{abstract}
RESUMEN: El presente artículo parte de la posibilidad de considerar la tragedia como un ejemplo general del proceso artístico, para comparar las relaciones básicas entre arte y naturaleza en la filosofía aristotélica. El objetivo central es probar que hay un principio de sustancialidad en la producción artística. La intención que subyace a esta investigación es encontrar fundamentos para explicar a qué puede referirse Aristóteles al decir que la tragedia y el arte tienen algo semejante a un principio vital, un alma.
\end{abstract}

PALABRAS CLAVE: Aristóteles, arte, narutaleza, producción, generación.
ABSTRACT: This article starts with the premise of conceiving tragedy as a general example of the artistic process in order to compare the fundamental relationship between art and nature in Aristotelian philosophy. Our main goal is to prove that substantiality exists in all artistic creations. The underlying purpose of this research is to discover the bases of Aristotle's argument that explain why both tragedy and art have something close to a vital principle, a soul.

KEYWORDS: Aristotle, art, nature, production, creation.

* Universidad Panamericana. 


\section{NATURALEZA Y PRODUCCIÓN: HACIA LA SUSTANCIALIDAD DE LA OBRA DE ARTE EN ARISTÓTELES}

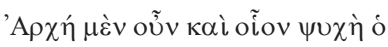
$\mu \tilde{\cup} \vartheta \varsigma \varsigma \tau \tilde{\eta} \varsigma \tau \rho \alpha \gamma \omega \delta i \alpha \varsigma^{\prime}$

\section{C} uando Aristóteles dice en el libro $a$ de la Metafísica que la verdad es fácil de encontrar, como cualquiera acierta a dar en una puerta, está aplicando una comparación claramente accidental. Nadie investigaría la naturaleza o la magnitud de las puertas para conocer qué es la verdad. Así, podría resultar absurdo estudiar en qué sentido puede Aristóteles hablar del alma en el contexto de la Poética, puesto que es obvio que la obra no es un ser animado y que el texto simplemente formula una comparación.

${ }^{1}$ Arist., Poética, 6, 1450a 38. La versión original del artículo presenta los textos escritos griego. He preferido transliterarlos aquí para facilitar su edición. Sigo para ello el modelo $A L A-L C$ publicado por R. K. Barry, Romanization Tables: Transliteration Schemes for Non-Roman Scripts. Library of Congress, 1997.
Sin embargo, la comparación que Aristóteles realiza entre el mito y el alma es mucho más radical que un mero ejemplo comparativo. Corresponde a una analogía que subraya los valores metafísicos propios de la Poética, según se puede comprobar en diversos textos de la obra aristotélica que señalan la primacía metafísica del mythos. Es el más importante de los elementos que conforman la naturaleza de la obra; ${ }^{2}$ es, junto con los hechos, el fin de la misma; ${ }^{3}$ sus partes constitutivas son las que actualizan la virtualidad de la poética; ${ }^{4}$ y los criterios más sólidos que Aristóteles orienta hacia

\footnotetext{
${ }^{2} P o, 6,1450$ a 15 y 1450 a 33.

${ }^{3} P_{0}, 6,1450$ a 23.

${ }^{4}$ Po, 6, 1450a 34 y 1450 b 19-20.
} 
la existencia de la obra son los relativos al mythos. ${ }^{5}$

Aristóteles no se refiere a que los mitos culturales sean el alma de las obras, como si éstas debieran construirse sobre las leyendas y el legado mítico de una sociedad concreta; a lo que aluden estos textos es a un valor intrínseco al hacer poético-mimético de acciones. En inglés, la palabra que traduce el concepto mythos es "plot", que hace referencia al argumento. Aristóteles dedica varias distinciones a la estructuración de acciones (systasis) y el mito a lo largo de los capítulos 6 al 14 de la Poética, como he señalado en otro lugar. ${ }^{6}$ La diferencia es sutil y consiste básicamente en las diferencias que hay entre la suma de las partes estructuradas entre sí (systasis) y el todo (mythos). ${ }^{7}$ Una forma clara de ejemplificar la diferencia está en la óptica con la que un artista se acerca a la obra, poniendo atención en los elementos que la conforman, la técnica, el estilo, los colores, el entramado en general: eso corresponde al principio como estructuración o systasis. ${ }^{8}$ En cambio, 25,30

${ }^{5}$ Po $, 7,1450 \mathrm{~b} 25,33 ; 1451$ a $10 ; 8,1451$ a

${ }^{6} \mathrm{C}$. Sabido, "Mito y estructuración en la Poética de Aristóteles", Iztapalapa n. 58, 2005, UAM, México, pp. 63-87.

${ }^{7}$ Po, $8,1451^{\text {a } 33-36 . ~ V e r ~ t a m b i e ́ n ~ R . ~ B i t t n e r, ~}$ "One Action" en A. O. Rorty (ed.), Essays on Aristotle Poetics, pp. 97-110.

${ }^{8}$ Cf. P. Ricoeur, Tiempo y narración I, 1987, Madrid, Cristiandad, pp. 85-6 y 102. el espectador se aproxima a la obra como un todo: no pone atención en la técnica de los actores (tratándose del cine, por señalar un género), la fotografía y la música. Simplemente deja que la obra sea y vive, convive con ella. Eso es propiamente el mythos.

El objetivo de este artículo es considerar la importancia metafísica del mythos y señalar una posible lectura metafísica del texto aristotélico en el que se encuentran algunas claves para comprender el ser de lo poético. Propongo en este trabajo que la afirmación "el mythos es como el alma" equivale a decir que corresponde a un sentido metafísico de la sustancia de la obra de arte.

Para desarrollar esta idea, el punto de partida es considerar al mythos desde la analogía entre la generación y la producción, con el fin de mostrar que es, en cierto modo, análogo a la physis o naturaleza. Profundizaré para ello en las analogías y comparaciones que Aristóteles desarrolla en los textos de Metafisica VII-7 y Física II. El carácter de principio de la physis, las diferentes instancias del proceso y el proyecto teleológico deben dar la clave para fundamentar la base de la analogía y considerar si es, efectivamente, aplicable a la mímesis como un tipo de producción.

A partir de estos razonamientos, será posible considerar la analogía 
NOTAS

del mythos con la sustancia. Su característica de fin, principio de unidad y acto del ser de la obra otorgan, además, validez a su carácter inteligible, de esencia y verosimilitud, como principio revelador de la realidad y verdad prácticas. De modo que se podrá afirmar que el mythos es el cumplimiento de la vocación poética y de su compromiso con la verdad práctica.

\section{Naturaleza y producción}

Antes de afirmar si, en efecto, el mythos conlleva en cierto modo la naturaleza de la obra, es necesario entender a qué sentido de naturaleza o physis se refiere esta conjetura. En la Física de Aristóteles se pueden hallar al menos unos diez significados distintos para el término physis. ${ }^{9}$ Estos se relacionan de una u otra manera, pero todos coinciden en que la naturaleza es un cierto modo de principio (archē) ${ }^{10}$

${ }^{9} \mathrm{Cf}$. R. Paniker, El concepto de naturaleza, 1951, Madrid, CSIC, p. 78.

${ }^{10}$ Aristóteles define "principio" (archē) en el libro V de la Metafísica como: "a) el punto de una cosa desde donde alguien puede comenzar a moverse; b) desde donde cada cosa puede hacerse del mejor modo; c) aquello desde lo cual, siendo intrínseco a la cosa, ésta comienza a hacerse; d) aquello desde lo cual, sin ser intrínseco a la cosa, ésta comienza a hacerse y desde donde principian naturalmente el movimiento del principio y el cambio; e) aquello según cuyo designio se mueven las cosas que se mueven y cambian las que cambian; $y, f)$ el punto desde donde una cosa empieza a ser cognoscible. Cf. Metaph., V-1, 1012b 34-1013a 17.
A todos los principios les es común ser lo primero desde lo cual algo es o se hace o se conoce. Y de éstos, unos son intrínsecos y otros extrínsecos. Por eso es principio la naturaleza, el elemento, la inteligencia, el designio, la sustancia y la causa final, pues el principio de conocimiento de muchas cosas es lo Bueno y lo Bello. ${ }^{11}$

De este texto se siguen tres modos generales de decir principio: "aquello desde lo cual algo es, o se hace o se conoce". E1 sentido que interesa particularmente aquí es el relativo a lo que se hace ${ }^{12}$ y que equivale al "principio de eficiencia" tanto en la génesis como en la poiēsis. ${ }^{13}$ Ahora bien, Aristóteles considera la physis como lo que "se hace" en dos aspectos: uno absoluto, que se aplica al paso del no ser al ser, al que se refieren los sentidos de "nacimiento", "principio engendrante" $\mathrm{y}$ "principio intrínseco de actividad". ${ }^{14}$ Otro en sentido relativo que se aplica de no

${ }^{11}$ Ibid., V-1, 1013a 17-23.

${ }^{12} \mathrm{E} 1$ verbo griego $\gamma \mathbf{i} \gamma v \mathrm{v} \mu \alpha \iota$, nacer, tiene en el contexto de la Física dos significados, según indica el traductor J. L. Calvo Martínez: (a) generarse, originarse, llegar al ser en sentido absoluto (aplōs); y, (b) convertirse en, tornarse, llegar a ser algo. Por ello se utiliza el sustantivo génesis en ambos sentidos. (Ph., I, n. 59). Cf. H. G. Liddell, y R.

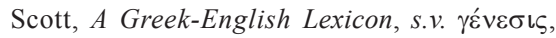

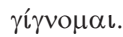

${ }^{13} \mathrm{Cf}$. A. González Álvarez, Tratado de metafisica, ontología, p. 415.

${ }^{14}$ Cf. W. D. Ross, Aristóteles, 1981, Buenos Aires, Charcas, trad. de D. F. Pró, pp. 98-9. 
ser a ser algo -que es propiamente el movimiento o cambio-, y se considera respecto de seres ya existentes que sufren alguna alteración. ${ }^{15}$

La physis, como aquello "desde lo que algo se hace", implica un sentido de actividad (que destaca el sufijo -sis) y de dinamismo. En el movimiento se manifiestan todos los sentidos de naturaleza. Por eso, Aristóteles advierte que toda consideración de "naturaleza", como principio, requiere, por un lado, analizar la relación entre las cuatro causas y, por otro, la relación entre la potencia y el acto. Cuando el análisis reduce los sentidos de la naturaleza a uno primero o más "general", Aristóteles concluye que physis puede tomarse ante todo como "forma", según el orden de las cosas, y como un "principio de operaciones", porque es por las operaciones como la generación y el nacimiento que conocemos la naturaleza. ${ }^{16}$ Esto implica un com-

${ }^{15} \mathrm{D}$. Bostock, "Aristotle on the Principles of Change in Physics I", Language and Logos, M. Schofield and M. Nussbaum (ed.), 1982, Cambridge, Cambridge University Press, pp. 180-1.

16 "Hay que tener en cuenta que la reducción de dos modos a uno puede hacerse de dos maneras. Una, según el orden de las cosas; otro, según el orden que se refiere a la imposición del nombre, pues nosotros ponemos los nombres según entendemos, porque los nombres son signo de lo inteligido. De ahí que, para nosotros, primero surja el nombre, el cual le compete a la cosa, sólo secundariamente, y así sucede en las decisiones, y porque las formas y las virtudes de las cosas se conocen por sus actividades, primero son la generación y el nacimiento los que reciben el nombre de natura- plejo de forma dinámica y agente intrínseco del movimiento, es decir que la naturaleza, desde la perspectiva del ser es "forma sustancial" y ante la perspectiva del cambio es "eficiencia". ${ }^{17}$

Los sentidos más propios de physis se aplican a lo que tiene este principio de operaciones de modo intrínseco. ${ }^{18} \mathrm{Su}$ contraste con el "arte" (téknē), que se desarrolla con constantes ejemplos en el libro segundo de la Física, consiste precisamente en la calidad intrínseca o extrínseca del principio del movimiento, es decir, si el objeto se mueve "por sí mismo" o "por otro". ${ }^{19}$ Aristóteles recurre a numerosos ejemplos de procesos técnicos para mostrar cómo opera la physis, de modo que él mismo considera la analogía entre arte y natura-

leza y, después, la forma. Pero según el orden de las cosas, primero le compete a la forma el nombre de naturaleza porque, como se ha dicho, de nada se dice que tenga naturaleza si no es por la forma", Comentario al libro $V$ de la Metafísica, trad. de Jorge Morán, nota 24.

${ }^{17}$ Cf. A. Ross, "La defensa aristotélica del uso de explicaciones teleológicas en Física II-8", Tópicos, 30bis, 2006, p. 139

${ }^{18}$ Cf. J. M. Cooper, "Aristotle on Natural Teleology", Language and Logos, en op. cit., pp. 198-9.

19 "Lo esencial es que el principio a quo del movimiento resida en el ser moviente-aquí engendrante-aunque el término ad quem recaiga en otro. Mis actos y acciones son naturales cuando surgen de mí mismo aunque recaigan en otro. Naturaleza implica principio intrínseco de acción, aunque ésta sea transeúnte. Principio intrínseco no implica acción inmanente." R. Paniker, op. cit., pp. 46-9. 
NOTAS

leza como "principio de operaciones" cuando no se trata de poner el acento en el origen de tal principio, sino en su modo de estar presente en la cosa, o, en este caso, en la realidad móvil. $^{20}$

Este modo de estar presente del principio de operaciones en la realidad es el modo de la forma, que visto desde cierto punto es intrínseco. En aquello que "cambia o se mueve", la forma no es un modo estático, sino dinámico. ${ }^{21}$ Es decir, que al considerar la naturaleza, hay una cierta relación formal en movimiento, que no pertenece a las formas accidentales o por concurrencia, ${ }^{22}$ sino a la forma

${ }^{20}$ Sobre este problema, se hace necesaria la distinción de lo que se hace "por naturaleza" y "según la naturaleza", según se tome en cuenta el origen del movimiento o el modo de proceder. En un caso, la diferencia se establece entre lo intrínseco y lo extrínseco. En el otro caso, según lo necesario o lo contingente. Lo natural en sentido estricto será entonces lo intrínseco y necesario, pero esta reducción no agota los sentidos de naturaleza. Cf. R. Paniker, ibid., pp. 52-5.

${ }_{21}$ "The form of any natural living kind consists of an interlocking and mutually supportive set of capacities, so that to explain the exercise of any one of these capacities by reference to the form is to link it to the further exercise of some other capacity for which it provides a supporting condition", J. M. Cooper, "Aristotle on Natural Teleology", p. 200.

${ }^{22}$ La traducción de la Física de Jose Luis Calvo, que cito con más frecuencia, traduce katá symbebēkós como "por concurrencia". Toma en cuenta que proviene del verbo symbainō y elige la traducción que significa "concurrir", "coincidir", "incidir". Concuerdo con Calvo en que "la traducción del latín per accidens llevada al castellano da inevitablemente una connotación de "azaroso" o sustancial. ${ }^{23}$ Esta razón de forma sustancial es una de las claves para comprender los tipos de cambio en Aristóteles y, entre ellos, la generación. Tanto la generación como la producción son un proceso en "camino hacia la naturaleza", es decir, hacia la presencia de esta forma sustancial. ${ }^{24}$

Aquello que nace, en tanto que nace, se dirige desde algo hacia algo. Ahora bien, ¿hacia qué nace? No hacia aquello "desde lo cual" sino "hacia lo cual". Por consiguiente, la naturaleza es la forma. ${ }^{25}$

Así pues, si bien puede decirse que lo producido se distingue de lo natural en que no tiene el principio por el cual llega a ser de modo "intrínseco", no puede decirse por ello que lo producido carezca de un principio de operaciones que, aunque tiene su

"casual" y que la palabra "accidente" tiene un significado un tanto luctuoso, que resulta negativo en la apreciación de los lectores". En cambio, la concurrencia hace referencia a una relación de elementos en realidades compuestas sin que su significado sea negativo o menos "puro" metafísicamente. Cf. J. L. Calvo, "Notas a la Física" núm. 20 y 34, pp. 8 y 13. Cf. H. G. Liddell, y R. Scott, A GreekEnglish Lexicon. s.v. $\sigma \cup \mu \beta \alpha i$ iv

${ }^{23} \mathrm{Cf}$. M. Artigas y J. J. Sanguineti, Filosofia de la Naturaleza, pp. 78-80. Hago notar que con frecuencia se llama a esta forma "propia de la sustancia" forma sustancial, aunque Aristóteles no se refiera explícitamente a ella. Le corresponde, sin embargo, lo que en Metafisica VII trata como especie.

${ }^{24} \mathrm{Ph}$., II, 193b 12-14.

${ }^{25} P$ h., II, 193b 16-18. Cf. W. D. Ross, "Commentary", Metaphysics, 1997, Oxford, Oxford University Press, pp. 294. 
origen en un agente extrínseco -el artista-, integra la obra con una "formalidad sustancial" existencial, que es principio de todas sus restantes concurrencias o "accidentes". ${ }^{26}$

Por eso distingue Aristóteles en la Física los términos "naturaleza", "por naturaleza" y "según la naturaleza". Naturaleza es un "cierto principio y causa del moverse o estar en reposo en aquello en que se da primariamente, por sí mismo". ${ }^{27}$ Todo lo que tiene "naturaleza", afirma Aristóteles, es "entidad, pues es algo que subyace y la naturaleza siempre reside en algo que subyace". ${ }^{28} \mathrm{El}$ origen de la naturaleza, es decir, el modo como ese principio llega a estar presente en la entidad puede ser exterior a sí mismo o estar en sí mismo pero no por sí mismo. ${ }^{29}$

Y lo mismo cada uno de los demás objetos que son fabricados: ninguno tiene en sí mismo el princi-

${ }^{26}$ "La forma substancial es la raíz de donde emana la estructura accidental de la sustancia. En este sentido, la forma es principio organizativo de la complejidad física del ente material, así como también principio dinámico, pues de ella brotan todas las actividades de la sustancia, todo lo que ésta hace en el ámbito material en que se mueva". M. Artigas y J. J. Sanguineti, Filosofía de la Naturaleza, p. 81.

${ }^{27} \mathrm{Ph}$., II, 192b 20.

${ }^{28} \mathrm{Ph}$., II, 192b 31-34.

${ }^{29}$ Este último es el que podría explicar una "naturaleza" presente en un artefacto o una obra de arte, que está presente en sí mismo, pero no por sí mismo. El artista es causa de su principio. Aristóteles trata con mayor detenimiento esta relación en Metaph., VII-9, 1034a 19-21. pio de la fabricación, sino que unas veces reside en otros y es exterior, como una casa y cada uno de los objetos manufacturados; otras veces, en cambio, está en ellos mismos aunque no por sí mismos, como cuantos podrían ser causas para sí mismos por concurrencia. ${ }^{30}$

De este principio -que es la esencia según la forma propia de la sustancia-se tiene conocimiento por sus operaciones. ${ }^{31}$ Aristóteles llama por eso a la naturaleza como principio de operaciones y distingue dos diferencias: ${ }^{32}$ por naturaleza (physei dè) significa aquello que tiene en sí mismo

${ }^{30} \mathrm{Ph}$., II, 192b 27-31. Aristóteles refiere aquí el famoso ejemplo del médico que se cura a sí mismo: "Un hombre, siendo médico, podría ser causante de salud para sí mismo; y sin embargo, no posee el arte de curar en tanto que se cura a sí mismo, sino que concurre incidentalmente el que la misma persona sea médico y se cure", $P h$., II, 192b 22-26. Así, un paciente tiene en sí el principio de la salud en cuanto a que puede mejorar y ser curado. De este modo, el médico que se cura a sí mismo lo hace, por un lado, gracias a su ciencia y por otro lado, porque naturalmente puede ser curado. El principio intrínseco de salud es éste que tiene "por ser hombre". El principio por concurren$c i a$ es el que tiene por ser médico. Por eso afirma Aristóteles que ambas cosas pueden darse por separado (192b 26), como ocurre entre el paciente que no sabe de medicina y el médico que no padece la enfermedad.

${ }^{31}$ Es una esencia dinámica, ya que le corresponde propiamente ser causa del movimiento en el ente móvil.

32 "En cambio, por naturaleza son estas y cuantas se dan en éstas por sí mismas, como el dirigirse hacia arriba se da en el fuego: esto no es "naturaleza", ni "tiene naturaleza", pero "es por naturaleza" y "conforme a naturaleza", $P h$., II, 192b 33-35. 
NOTAS

o en algo idéntico a sí mismo el agente del movimiento: "es todo cuanto, moviéndose desde un cierto principio, que tiene en si mismo, alcanza un cierto fin". ${ }^{33}$ En cambio, según la naturaleza (katà physin) se refiere a aquello que tiene un principio según el cual opera, sea su procedencia intrínseca o extrínseca, con miras a una finalidad. ${ }^{34}$

Por ejemplo, si una casa fuera de las cosas que se producen por naturaleza, se produciría igual que ahora con la intervención del arte. Y si las cosas debidas a la Naturaleza no se originaran sólo por Naturaleza, sino también por arte, se originarían de la misma forma en que son por naturaleza. Luego una cosa es para la otra. En general, el arte en unos casos lleva a término lo que la Naturaleza es incapaz de completar y en otros la imita. Por consiguiente, si los objetos del arte son para algo, es evidente que también lo son los de la Naturaleza. ${ }^{35}$

Principalmente, en este aspecto se puede basar la analogía entre arte y naturaleza, como un proceso eficiente con miras a una finalidad. Aristóteles recurre a los ejemplos del arte para distinguir las causas extrínsecas de las intrínsecas porque

${ }^{33} \mathrm{Ph}$., II, 199b 15-17.

${ }^{34}$ J. M. Cooper, "Aristotle on Natural Teleology", p. 206.

${ }^{35} \mathrm{Ph}$., II, 199a 12-20. en las cosas naturales, que tienen el principio de "naturaleza" en sí mismas y actúan "por naturaleza", esta diferencia es más difícil de ver. ${ }^{36}$ Sin embargo, si Aristóteles recurre a este ejemplo es porque en ambos casos el proceso tiene una semejanza en su orden formal dinámico, en cuanto a la relación entre la naturaleza como causa eficiente y la finalidad, como aquello "hacia lo cual" se tiende con la actividad. ${ }^{37}$

Cuando se plantea la posibilidad de que haya una "naturaleza" en la obra de arte, ésta debe corresponder, por lo tanto, al principio de operaciones intrínseco a ella, aunque ciertamente no sea apropiado hablar de naturaleza en cuanto al "origen" de tal

${ }^{36}$ Las causas que en el análisis pueden separarse, en la realidad se asimilan. Ver también J. Morán, "Evidencia de la naturaleza en Aristóteles", Tópicos 6 (1994), pp. 76-7. Cabe destacar que, al conocer el proceso de producción de la obra, de algún modo se conoce mejor su estructura, por connaturalidad. En el proceso que va del conocer lo más evidente a nosotros a lo más evidente en sí mismo, la con naturalidad que implica el conocimiento del proceso productivo es relevante.

37 "La forma es el plan de una estructura considerada como informando un producto particular de la naturaleza o el arte. La causa final es el mismo plan, considerado como no todavía realizado en la cosa particular, sino hacia lo que se dirige la naturaleza o el arte [...] La causa final en el arte es, así, en sentido estricto, una cierta estructura que el artista se esfuerza conscientemente por incorporar a una materia particular. La causa final en la naturaleza es la estructura común a toda una infima species, a la cual tienden todos los miembros individuales de una especie, sin ningún propósito consciente de renovar su estructura individual". W. D. Ross, op. cit., pp. 110-111. 
principio. ${ }^{38}$ Por eso conviene entender cómo se establece la relación entre la causa eficiente y la final, en general, para todas las realidades móviles (que admiten cambio). Ello implica reflexionar sobre las teorías de Aristóteles acerca del cambio (metabolē) $y$, en particular, lo que se refiere a la generación y la producción. ${ }^{39}$

\section{Metabolē y producción}

Ahora es necesario estudiar qué tipo de proceso es la generación para Aristóteles; sólo a partir de las características específicas de la generación como cambio (metabolē) se puede fundamentar una analogía en el proceso de la producción. Si esta analogía fuese posible, podría decirse también que el principio que causa la producción es análogo a la naturaleza. ${ }^{40}$ Así, habría un principio según la naturaleza que opera en la producción de

${ }^{38}$ Cf. W. D. Ross, op. cit., p. 190-1. También puede considerarse la "causa formal extrínseca", como en el caso de la casa que está en la mente del arquitecto cuando la construye. El problema de la "causa ejemplar" de la que se ha hablado ya al hablar de la mímésis es precisamente que la causa formal (el proyecto en la mente del arquitecto) y la causa eficiente (el arquitecto en cuanto tal) se relacionan de un modo peculiar cuando se pone la potencia operativa poética en acto y constituye su téknē. Así, la tékne en el pasaje de la Metafísica corresponde a la causa formal extrínseca que corresponde también a la causa eficiente.

${ }^{39}$ Cf. W. D. Ross, op. cit., p. 192.

${ }^{40} \mathrm{En}$ este caso, la naturaleza del engendrante. un modo semejante a cómo opera la naturaleza en la generación. ${ }^{41}$ Se trata entonces de pensar en "dos naturalezas": por un lado, la que opera en el llegar a ser de la obra y por otro, de un modo análogo, la que constituye forma sustancial de la obra. En última instancia, lo que se quiere demostrar es que la mímēsis como actividad del poeta equivale a la naturaleza del engendrante y el mythos a la esencia de la obra de arte.

Dice Aristóteles en el libro III de la Física, que la physis es un principio de movimiento (kínēsis) y de cambio (metabolē) ${ }^{42}$ Aunque Aristóteles utiliza los términos kínēsis y metabole indistintamente en ciertos contextos de la Física, es indispensable precisar la diferencia entre uno y otro. En sentido amplio, ambas palabras con respecto a la physis implican una "modificación". ${ }^{43}$ Sin embargo, metabolē responde a un sentido amplio del que la kínēsis es sólo un modo. Metabolē es la modificación propia de los cambios "intrínsecos", y kínēsis se aplica de modo especial a modificaciones "extrínsecas" que ocurren a la sustancia sin corromperla, como el cambio de lugar, tamaño y cualidad. Las distintas kinēseis también son metabolè en cierto senti-

${ }^{41}$ Cf. Ph., II-8, 199a 15-19.

${ }^{42} \mathrm{Ph}$., III-1, 200b 12.

${ }^{43}$ Cf. W. D. Ross, op. cit., pp. 121-2 y núm. 67. 
do, de modo que todo movimiento implica un tipo de cambio, pero no todo cambio es propiamente un movimiento. ${ }^{44}$

En este sentido amplio de "modificación" (metabolē) define Aristóteles el movimiento como "actualización de lo que está en potencia en tanto que está en potencia". ${ }^{45}$ El impasse entre la potencia y su actualización en el devenir permite pensar en la acción constante del principio durante el proceso. ${ }^{46}$ Por otro lado, es importante señalar la distinción que apunta Ross entre movimiento y actividad en este sentido: el movimiento es la actividad incompleta y la actividad (enérgeia) es el movimiento comple-

${ }^{44}$ Como señala Bostock, el tratado de la Física, que se plantea primero como una investigación sobre los objetos de la realidad natural, deriva en la pregunta por sus principios. Del mismo modo, no sólo se pregunta por los principios del cambio natural (animado, inanimado, natural o artificial) sino por los principios de todo cambio. Cf. D. Bostock, "Aristotle on the Principles of Change in Physics I", pp. 182-3.

${ }^{45} \mathrm{Ph}$., III-1, 201b 5. Tomás de Aquino parafrasea esta definición como "modo medio entre la potencia y el acto", ya que el solo acto es el término del movimiento y la potencia es anterior al movimiento. Cf. Tomás de Aquino, Comentario a la Física de Aristóteles, II-2, n. 194.

46 "Sólo cuando la construcción está haciéndose, lo edificable como tal se está actualizando, y la construcción es precisamente su actualización. [...] Así, forma parte de la naturaleza del movimiento el hecho de que lo potencial no haya perdido todavía completamente su potencialidad y se haya convertido en devenir actual; esa es la diferencia entre movimiento y actividad". W. D. Ross, op. cit., p. 121. tado. ${ }^{47}$ Lo que está en juego en este contexto es la posibilidad de fundamentar una relación que vaya desde el agente del movimiento (la actividad del poeta) hasta la constitución de un principio sustancial del producto (la actualidad de la obra de arte).

El movimiento, como actualización de una potencia, requiere siempre de un agente que ponga en acto dicha capacidad o potencia. ${ }^{48}$

También es evidente esto que produce perplejidad: que el movimiento se da en lo que es movible, pues es la actualización de esto, pero por agencia de aquello que es capaz de mover. Sin embargo, la actualización de lo que mueve no es diferen-

${ }^{47}$ Esta diferencia señala un dato importante cuando se tiene en cuenta la dimensión de la mímēsis como actividad, así como el desarrollo de la poiēsis. No queda del todo clara la diferencia entre ambas actividades cuando se trata del proceso, si bien es cierto que la mimésis como tal permanece en todo el proceso y fundamenta el mythos como la entelékeia o la realización final de la enérgeia, mientras la poíesis parece responder más bien a la kinēsis, al proceso en movimiento. Cf. R. Paniker, op. cit., p. 270 .

${ }^{48} \mathrm{El}$ agente del movimiento corresponde a la "causa que mueve". Tradicionalmente se le denomina causa eficiente y suele considerarse una causa extrínseca per se. No obstante, Aristóteles admite que existen entre las realidades móviles aquellas cuya causa agente es intrínseca, como en el caso de los vivientes, y operan por "automoción”. (Aún así, en el orden interno de sus partes, una parte mueve a la otra). En el caso de la naturaleza como principio de operaciones, puede ser la propia naturaleza el principio agente del movimiento; esto ocurre de modo especial en los procesos naturales, como la generación. Cf. J. M. Cooper, “Aristotle on Natural Teleology", pp. 201-2. 
te, pues tiene que ser la actualización de ambos: ello es capaz de mover en virtud de la potencia, pero mueve de hecho en virtud de la actualización. Con que es actualizador de lo movible de modo que la actualización de ambos es una y la misma. ${ }^{49}$

En el problema de los tipos de cambio es importante distinguir los cambios cuya causa agente es intrínseca o extrínseca, debido a la relación de actualidad que hay entre el acto del agente del movimiento y el acto de lo movido. ${ }^{50}$ Esta diferencia es importante porque, vista desde un cierto aspecto, al ser el poeta un agente externo, puede pensarse que no hay propiamente una relación de actualidad con lo producido. Sin embargo, hay que ver si el principio del cambio en la producción da lugar a una cierta "forma" o principio de operaciones, aun cuando no se trate de un proceso de automoción, donde el acto agente es intrínseco, sino un proceso productivo con un agente extrínseco -el poeta- y con una naturaleza distinta del acto intrínseco en la obra producida. La naturaleza de la obra no es idéntica a la naturaleza

${ }^{49} P h$., III-3, 202a 13-18.

${ }^{50}$ Es decir, entre el acto que da lugar al movimiento y la actualización de lo movido, la enérgeia. Cf. R. Paniker, op. cit., pp. 272-3. Este tipo de unidad es semejante al que se expone en De Anima III-2, 425b 27, concerniente a la unión entre el sensible y el sentido en acto. del poeta. ${ }^{51}$ Lo importante, en todo caso, es reconocer qué tipo de relación hay entre la actividad del poeta y el acto de la obra que ha producido.

Para Aristóteles hay dos tipos de cambio: el cambio sustancial y el cambio accidental. ${ }^{52}$ Esta división surge a partir de los cuatro géneros de metabole: la alteración, el aumento y la disminución, la traslación y la generación y corrupción. ${ }^{53}$ Son movimientos (kinēsis), propiamente, los tres primeros géneros que corresponden a los accidentes de cualidad, cantidad y lugar. ${ }^{54}$ El cambio sustancial es en el cual el resultado de la modificación es que la sustancia deja de ser la que era y se transforma en otra sustancia distinta, o que del no ser

${ }^{51}$ El problema puede plantearse así: ¿la unidad entre el acto del agente y el acto de lo movido puede dar lugar a un acto intrínseco en lo movido? Por ejemplo, el que yo lea un libro no implica que, una vez realizada la actividad, a partir de la correspondencia de mi acto con su potencialidad de ser leído, dé como resultado que el libro pueda leerse a sí mismo. Cf. M. Artigas y J. J. Sanguineti, Filosofia de la Naturaleza, pp. 159-60. En todo caso, este problema se resuelve con el concepto de potencia activa.

${ }^{52}$ La división entre cambio sustancial y accidental no es explícitamente aristotélico, pero no deja de ser una síntesis congruente. La razón es complicada y se expone en diversos libros de la Física (III, V y VIII-3), de la Metafisica (VII, X y XI) y en el Tratado acerca de la Generación y la Corrupción (I-4). El problema consiste en que aun compartiendo los principios básicos del proceso de metabolē, la generación y producción no son estrictamente un movimiento o kínēsis. Cf. W. D. Ross, op. cit., p. 122.

${ }^{53}$ Cf. $P$ h., III-1, 201a 11 a 16, esp. 14-15.

${ }^{54}$ Cf. Metaph., XI-12, 1068 b 16-20. 
NOTAS

llega a ser, o que de ser, llega al no ser: en este caso se encuentran la generación y la corrupción. El cambio accidental es el que sufre una sustancia que, permaneciendo, padece ciertas modificaciones cualitativas, cuantitativas o locales, como los desplazamientos, los cambios de color, el crecimiento o la disminución. Así, por ejemplo, la adquisición de un hábito es un cambio accidental, y la muerte un cambio sustancial.

$\mathrm{Si}$, en efecto, hay una analogía entre la generación y la producción (en general y poética en particular), entonces producir debe ser también un cambio sustancial, cuyo agente productor es extrínseco, pero al actualizar el principio del movimiento imprime la forma sustancial en una materia, produce un cambio intrínseco y mueve hacia la existencia de una sustancia individual, en cierto modo puede decirse que nueva, cuyo principio, una vez existente, será también un cierto modo de naturaleza. ${ }^{55}$

\section{El proceso de generación}

La generación es una metabolē, un proceso de cambio que implica tiempo, lugar y magnitud. Involucra la interacción de las cuatro causas y la

${ }^{55}$ Cf. W. D. Ross, "Commentary” Metaphysics, pp. 182-183. perfección de la potencia en acto. Lo que en la Física reúne a la naturaleza con la generación y la producción es, ante todo, el esquema del proceso teleológico, donde se equiparan naturaleza, arte y pensamiento. ${ }^{56} \mathrm{En}$ el libro VII de la Metafisica Aristóteles afirma:

De las cosas que se generan, unas se generan por naturaleza, otras por arte y otras espontáneamente. Y todas las que se generan llegan a ser por obra de algo y desde algo y algo. ${ }^{57}$

En esta cita se incluye, dentro de la generación, lo que tiene como causa la naturaleza en el sentido de principio eficiente intrínseco; el arte, en el sentido de causa eficiente extrínseca y lo espontáneo, en el sentido de lo casual (ya porque se desconozca la causa o porque no exista en ella una relación entre la causa eficiente y la final). ${ }^{58}$ Esta inclusión general de naturaleza, arte y azar dentro de las causas de la generación responde a que Aristóteles estudia en este libro el proceso de la generación desde una perspectiva abierta. Responde, como se ha dicho, al planteamiento teleológico, donde lo que más interesa es el

${ }^{56} \mathrm{Cf}$. Tomás de Aquino, op. cit., I-13, n. 78 y III-2, nn. 194-195.

${ }^{57}$ Metaph., VII-7, 1032a 12-14.

${ }^{58} \mathrm{Cf}$. Tomás de Aquino, ibid., VIII-7, n. 791 
vínculo entre la forma que imprime el agente y el fin del proceso. ${ }^{59}$

Lo que se genera llega a ser "por obra de algo", es decir, de una acción eficiente, "desde algo" que es el sustrato del cambio, $y$ "algo" que es el ser que resulta al final del proceso. En las realidades naturales hay una cierta "homonimia" entre la forma y la eficiencia. Digo homonimia y no identidad por la distancia real que hay entre la forma del engendrante y el engendrado. ${ }^{60} \mathrm{La}$ forma que pasa del engendrante al engendrado es igual en cuanto a su esencia, pero no es la misma y no se destruye el engendrante al engendrar, de modo que es, en cierto modo, una actividad transitiva.

Si el agente es la naturaleza, (por un orden dado o por automoción en el caso de un ser vivo, guiado por una tendencia necesaria) o el pensamiento (por la deliberación ante lo contingente), o el arte, lo que importa en este caso es que el agente imprime una forma. ${ }^{61}$ Por eso incluye Aristóteles al azar y lo espontáneo en esta consideración. Si fuera del orden de la naturaleza o del que establece la razón, algo modifica o imprime una forma en la materia (y puede

${ }^{59}$ Cf. J. M. Cooper, op. cit., pp. 200-1.

${ }^{60} \mathrm{Cf}$. W. D. Ross, op. cit., pp. 191-2.

${ }^{61} \mathrm{Y}$ no una forma cualquiera, sino una "forma sustancial". Es decir, aquel acto que da el ser al compuesto. Cf. M. Artigas y J. J. Sanguineti, op. cit., p. 88. suceder debido a la condición potencial de la materia como sustrato del cambio), ese algo indeterminado es también, en cierto modo, un agente. ${ }^{62}$

De hecho, la condición de agente del azar queda reafirmada por la figura de la tychē o fortuna, porque una vez acaecido lo espontáneo, el "pensamiento" puede asimilarlo y apropiarse del curso que imprime. Así, es afortunado lo que ocurre espontáneamente, pero tal como se hubiese querido, y desafortunado lo que espontáneamente sucede y resulta contrario a lo que se podría querer. ${ }^{63}$ Lo que sucede entonces es que lo azaroso es integrado al orden por la razón. No sobra esta precisión aristotélica en el tema de la actividad artística, porque la relación de la actividad poética con la materia sensible es inevitable. Con frecuencia, un suceso afortunado contribuye a la perfección de la obra: una veta, una sombra, un color, una palabra. No resta la labor del pensamiento, que da orden a la realidad, antes bien subraya la flexibilidad con que opera la actividad poética.

${ }^{62} \mathrm{Cf}$. Tomás de Aquino, op. cit., II-10 n. 156.

${ }^{63}$ Es curioso que Aristóteles explique de un modo tan confuso un asunto tan cotidiano. La redacción del ejemplo siempre es complicada aunque el suceso es simple: hallarse a alguien de camino hacia otro lado; alguien que te debe dinero justo cuando vas a cobrar. Este tipo de acontecimientos, con mayor o menor trascendencia, se suceden en la vida diaria. Cf. Ph., II-6, 197b 14-35. 
CITAM Derechos Reservados.

La reproducción total o parcial de este artículo se podrá hacer si el ITAM otorga la autorización previamente por escrito. 